\title{
Clinical outcomes and surgical preferences for breast-conserving surgery and mastectomy: a propensity score-matched analysis
}

\author{
Mawin Vongsaisuwon', Krit Pongpirul ${ }^{2,3, *}$, Kris Chatamara ${ }^{4}$
}

\begin{abstract}
Background: While numerous randomized controlled trials have demonstrated long-term survival rates for patients with early-stage breast cancer treated with breast-conserving surgery (BCS) comparable to mastectomy, the latter remains the most prevalent surgical option to treat early-stage breast cancer in Thailand.

Objectives: To investigate the potential determinants affecting the decision on selecting BCS or mastectomy for the treatment of early-stage breast cancer and to compare the disease-free survival and overall survival between the treatments using a propensity score-matched analysis.

Methods: Patients diagnosed nonmetastatic breast cancer at the Queen Sirikit Breast Cancer Center from January 2006 to December 2015, were retrospectively identified and grouped intro patients who received BCS or mastectomy. After propensity score matching, 356 BCS and 209 mastectomy patients were identified, and statistical analysis was conducted to determine treatment selection factors and compare disease-free and overall survival.

Results: Disease-free survival and overall survival in months comparing BCS and mastectomy were not statistically different with $P$ values of 0.11 and 0.77 , respectively. Determinants of treatment selection found that younger age, surgeon preference, smaller tumor size, and lower tumor grade were statistically significant factors in the selection of BCS over mastectomy. The majority of surgeons had a preference for one treatment over the other $(P<0.001)$.

Conclusion: The outcome of BCS is comparable to mastectomy in early-stage breast cancer patients. Key determinants affecting the selection of treatment were identified to be patient age, characteristics of the tumor, and surgeon's preference.
\end{abstract}

Keywords: breast-conserving surgery; mastectomy; retrospective study

Breast cancer is the leading cause of cancer-related deaths in women, with global trends indicating rising rates of incidence and mortality [1]. Breast-conserving surgery (BCS) is the removal of cancerous tissue followed by radiation treatment, allowing the maintenance of healthy breast tissue in breast cancer patients. BCS is becoming a preferred treatment approach for early-stage breast cancer, yet has not replaced mastectomy. Although numerous randomized controlled trials have demonstrated long-term survival rates for patients with early-stage breast cancer treated with BCS comparable to

*Correspondence to: Krit Pongpirul, Department of Preventive and Social Medicine, Faculty of Medicine, Chulalongkorn University, Bangkok 10330, Thailand, e-mail: krit.po@chula.ac.th

'Department of Surgery, Faculty of Medicine, Chulalongkorn University, Bangkok 10330, Thailand

2Department of Preventive and Social Medicine, Faculty of Medicine, Chulalongkorn University, Bangkok 10330, Thailand

${ }^{3}$ Department of International Health, Johns Hopkins Bloomberg School of Public Health, MD 21205, USA

${ }^{4}$ Queen Sirikit Centre for Breast Cancer, King Chulalongkorn Memorial Hospital, Bangkok 10330, Thailand

O Open Access. (C) 2019 Mawin Vongsaisuwon et al., published by Sciendo. (c))BY-NC-ND This work is licensed under the Creative Commons Attribution NonCommercial-NoDerivatives 4.0 License. 
mastectomy, the latter remains the most prevalent surgical option to treat early-stage breast cancer in Thailand [2-7]. The preference for mastectomy over BCS may be a result of surgeon skills and preferences as well as a lack of facilities and confidence in the treatment itself $[8,9]$.

Currently, no studies have attempted to compare the outcomes of BCS to mastectomy in a real-life setting, where the selection of treatment takes into account numerous factors. The three key determinants in the decision making of the treatment include disease, patient and physician factors. Disease factors include the stage, extent, and molecular subtype of cancer, with mastectomy being the treatment of choice for locally advanced cancer stages and multifocal or multicentric diseases, while BCS has a greater chance of selection in HER-2 molecular subtypes due to the associated multifocal and multicentric extent of disease. Patient factors include patient preference, with BCS being the preferred treatment option in younger patients, and underlying disease as patients with certain underlying conditions may not be the candidates for BCS, such as active systemic lupus erythematosus, which prevent patients from being treated with radiation postsurgery. Physician factors include the skills and preference of the surgeon as well as the accessibility to radiotherapy [10].

Propensity score-matched analysis is commonly used to reduce bias from observational retrospective studies, as the analysis accounts for numerous factors on the effects and outcomes of treatments. The selection of treatment for early-stage breast cancer is affected by many factors; therefore, a propensity score-matched analysis can be used to reduce the bias on results of treatment outcomes. This study aimed to investigate the potential determinants affecting the decision on selecting BCS or mastectomy for the treatment of early-stage breast cancer and to compare the disease-free survival and overall survival between the treatments using a propensity scorematched analysis.

\section{Materials and methods}

The study was conducted at King Chulalongkorn Memorial Hospital (KCMH), one of the largest hospitals in Bangkok with an in-patient capacity of 1,435 beds. The hospital, operated by the Thai Red Cross Society, serves as the teaching hospital for the Faculty of Medicine, Chulalongkorn University. Patients diagnosed with early-stage breast cancer and treated by either BCS or mastectomy at the Queen Sirikit Breast Cancer Center, KCMH from January 2006 to December 2015, were retrospectively identified. Patients diagnosed with cancer stage 0 to III breast cancer from the Queen Sirikit cancer registry were included in the study. All procedures performed in this retrospective cohort study were in accordance with the ethical standards of the Institutional Review Board, Faculty of Medicine, Chulalongkorn University (certificate of approval no. 234/58) and with the 1964 Helsinki declaration and its later amendments or comparable ethical standards.

Data obtained from the Queen Sirikit Cancer Registry were verified for accuracy by Vongsaisuwon M. Patient details, including age and menopausal status, the name of the consulting breast surgeon and details on the diagnosis, and treatment were collected. Tumor characteristics, including morphology, tumor grade, estrogen receptor, progesterone receptor, and human epidermal growth factor receptor 2 expression, were recorded. The cancer stage at diagnosis, including details on tumor size, nodal status, and presence of metastases as well as the dates of diagnosis and recurrences was also noted. Additional data from comprehensive electronic medical records (EMR) was obtained, including information on clinical encounters, diagnosis, prescribed medications, laboratory results, and pathology reports.

Patients were grouped by the type of definitive surgery chosen for the treatment. Surgical designation of lumpectomy, excisional biopsy, partial mastectomy, re-wide excision, and segmental mastectomy was considered BCS. While surgical designations of mastectomy, modified radical mastectomy, radical mastectomy, subcutaneous mastectomy and total (simple) mastectomy, skin-sparing mastectomy, all with or without implant and reconstruction, were considered mastectomy.

Descriptive statistics were carried out and Student's $t$-test, Chi-Square, or Fisher's exact test were applied as appropriate. The clinical outcomes of local recurrence and death comparing patients who underwent mastectomy and BCS were adjusted for age, surgeon, hormonal status, HER-2 status, tumor grade, clinical size, and pathology stage. Kaplan-Meier estimates of patient disease-free survival and overall survival were analyzed using the log-rank test. Propensity score matching was applied to reduce potential bias due to confounding variables. Statistical analysis was performed using the Stata/MP version 15 (StataCorp LLC, College Station, TX).

\section{Results}

\section{Baseline characteristics}

Among the 714 nonmetastatic breast cancer patients identified from the Queen Sirikit Cancer Registry, 260 underwent mastectomy, while 454 underwent BCS (Figure 1). The results on the determinants of treatment selection found that there were significant differences in the patient age, surgeon, 


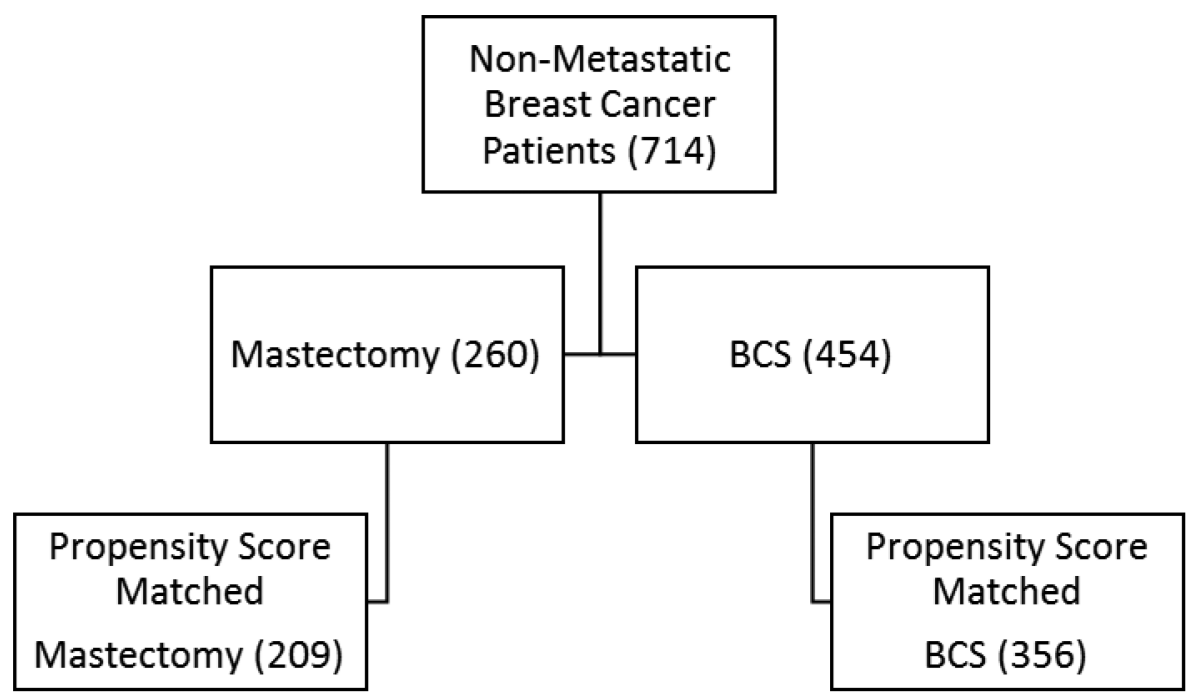

Figure 1. Flow diagram of the study sample.

clinical tumor size, tumor grade, tumor age, while there were no significant differences in menopausal status, hormonal status positive, and HER-2 positive (Table 1). Patient age had a significant impact on the treatment selection, as BCS was more common in patients aged 31 to 70 years, while mastectomy was the preferred treatment for patients aged 71 years or greater $(P=0.010)$. The majority of surgeons had a preference for one treatment over the other, Surgeons A, B, and D performed $\mathrm{BCS}$ in $62 \%, 76 \%$, and $70 \%$ of their patients, respectively, while Surgeon C and E performed mastectomy in $73 \%$ and $64 \%$ of their patients, respectively $(P<0.001)$. Patients who had undergone BCS had a smaller average clinical tumor size $(P<0.001)$ and were diagnosed with earlier stages of cancer $(P=0.002)$ compared to mastectomy patients, $1.48 \mathrm{~cm}$ at stages $0-1$ and $2.45 \mathrm{~cm}$ at stages $2-3$, respectively. Although more patients with all grades of tumors underwent $\mathrm{BCS}$, there was a decreasing preference for BCS with higher tumor grades $(P<0.001)$. The distribution of tumor stages was comparable across surgeons $(P=0.358)$.

Based on the comparison of baseline characteristics, the results found that treatment outcomes of death or recurrence of cancer between mastectomy and BCS groups were also not statistically different, with $P$ values of 0.877 and 0.825 , respectively.

\section{Propensity score matching}

A propensity score accounts for all other factors that may confound the results in determining the probability of selecting a treatment due to a specific factor. With propensity score matching, taking into account all baseline
Table 1. Baseline characteristics

\begin{tabular}{|c|c|c|c|}
\hline & Mastectomy $(n=260)$ & $\operatorname{BCS}(n=454)$ & $P$ \\
\hline Age & & & 0.010 \\
\hline $21-30$ & $3(1.15 \%)$ & $3(0.66 \%)$ & \\
\hline $31-40$ & 21 (8.08\%) & 41 (9.03\%) & \\
\hline $41-50$ & $69(26.54 \%)$ & 167 (36.78\%) & \\
\hline $51-60$ & $93(35.77 \%)$ & $143(31.50 \%)$ & \\
\hline $61-70$ & 42 (16.15\%) & 75 (16.52\%) & \\
\hline $71-80$ & $26(10.00 \%)$ & $22(4.85 \%)$ & \\
\hline $81-90$ & $5(1.92 \%)$ & $3(0.66 \%)$ & \\
\hline $91-100$ & $1(0.38 \%)$ & $0(0.00 \%)$ & \\
\hline Menopause & 165 & 259 & 0.097 \\
\hline Surgeon & & & $<0.001$ \\
\hline A & $127(48.85 \%)$ & 209 (46.04\%) & \\
\hline B & $56(21.54 \%)$ & $178(39.21 \%)$ & \\
\hline $\mathrm{C}$ & 47 (18.08\%) & $17(3.74 \%)$ & \\
\hline $\mathrm{D}$ & $18(6.92 \%)$ & $42(9.25 \%)$ & \\
\hline $\mathrm{E}$ & $9(3.46 \%)$ & $5(1.10 \%)$ & \\
\hline $\mathrm{F}$ & $3(1.15 \%)$ & $3(0.66 \%)$ & \\
\hline $\begin{array}{l}\text { Avg. clinical tumor } \\
\text { size }(\mathrm{cm})\end{array}$ & 2.45 & 1.48 & $<0.001$ \\
\hline $\begin{array}{l}\text { Hormonal status } \\
\text { positive }\end{array}$ & 189 & 339 & 0.554 \\
\hline HER-2 positive & 51 & 77 & 0.133 \\
\hline Tumor grade & & & $<0.001$ \\
\hline 1 & $32(12.31 \%)$ & $113(24.89 \%)$ & \\
\hline 2 & $106(40.77 \%)$ & $171(37.67 \%)$ & \\
\hline 3 & $86(33.08 \%)$ & $130(28.63 \%)$ & \\
\hline Unknown & 36 (13.85\%) & 40 (8.81\%) & \\
\hline
\end{tabular}


Table 1. Baseline characteristics (continued)

\begin{tabular}{cccc}
\hline & Mastectomy $(\mathbf{n}=\mathbf{2 6 0})$ & BCS (n= 454) & $\boldsymbol{P}$ \\
\hline Tumor stage & & & $<0.001$ \\
0 & $21(8.08 \%)$ & $49(10.79 \%)$ & \\
1 & $49(18.85 \%)$ & $204(44.93 \%)$ & \\
2 & $132(50.77 \%)$ & $170(37.44 \%)$ & \\
3 & $56(21.54 \%)$ & $28(6.17 \%)$ & \\
Unknown & $2(0.77 \%)$ & $3(0.66 \%)$ & \\
Recurrence & 35 & 63 & 0.877 \\
Death & 10 & 16 & 0.825 \\
\hline
\end{tabular}

characteristics such as age, menopausal status, surgeon, and clinical tumor size, 209 mastectomy patients and 356 BCS patients were included (Figure 1). The statistical significance of each covariate in selecting BCS or mastectomy from the propensity score-matched results is less biased compared to results without propensity score matching, due to the unmatched confounding factors, which lead to higher variances in the sample (Figure 2).

With propensity score matching, the results on the determinants of treatment selection found that patient age, surgeon, clinical tumor size, and tumor grade were all statistically significant factors in the selection of treatment, while menopausal status, hormonal status positive, and HER-2 positive were not statistically significant factors (Table 2). Patients aged $<70$ years were commonly treated by BCS, while patients more than 71 were treated by mastectomy $(P=0.005)$. All surgeons had a clear preference for one treatment over the other, Surgeons $\mathrm{A}, \mathrm{B}, \mathrm{D}$, and $\mathrm{F}$ performed $\mathrm{BCS}$ in $63 \%, 77 \%, 71 \%$, and $60 \%$ of their patients, respectively, while Surgeons $C$ and $E$ performed mastectomy in $75 \%$ and $64 \%$ of their patients, respectively $(P=0.001)$. The average clinical tumor size in
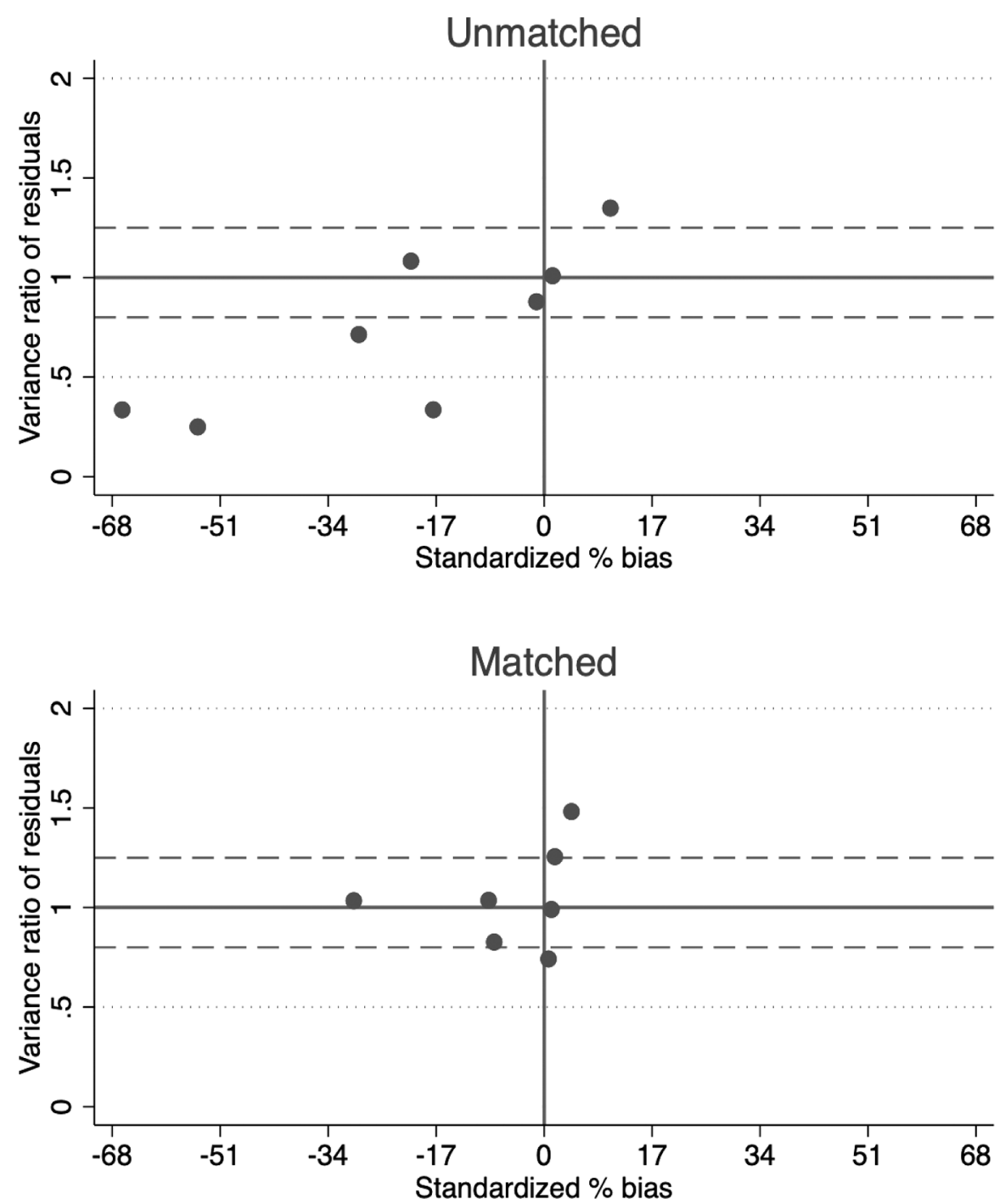

Figure 2. Residual variance in unmatched and matched samples. 
Table 2. Propensity score matching

\begin{tabular}{|c|c|c|c|}
\hline & Mastectomy $(n=209)$ & $\operatorname{BCS}(n=356)$ & $P$ \\
\hline Age & & & 0.005 \\
\hline $21-30$ & $2(0.96 \%)$ & $3(0.84 \%)$ & \\
\hline $31-40$ & 20 (9.57\%) & 35 (9.83\%) & \\
\hline $41-50$ & $50(23.92 \%)$ & $131(36.80 \%)$ & \\
\hline $51-60$ & $76(36.36 \%)$ & $110(30.90 \%)$ & \\
\hline $61-70$ & $36(17.22 \%)$ & $61(17.13 \%)$ & \\
\hline $71-80$ & $21(10.05 \%)$ & $14(3.93 \%)$ & \\
\hline $81-90$ & $3(1.44 \%)$ & $2(0.56 \%)$ & \\
\hline $91-100$ & $1(0.48 \%)$ & $0(0.00 \%)$ & \\
\hline Menopause & 137 & 196 & 0.021 \\
\hline Surgeon & & & 0.001 \\
\hline$A$ & 99 (47.37\%) & $171(48.03 \%)$ & \\
\hline B & $38(18.18 \%)$ & $126(35.39 \%)$ & \\
\hline C & 46 (22.01\%) & $15(4.21 \%)$ & \\
\hline $\mathrm{D}$ & $15(7.18 \%)$ & $36(10.11 \%)$ & \\
\hline $\mathrm{E}$ & $9(4.31 \%)$ & $5(1.40 \%)$ & \\
\hline $\mathrm{F}$ & $2(0.96 \%)$ & $3(0.84 \%)$ & \\
\hline $\begin{array}{l}\text { Clinical tumor size } \\
(\mathrm{cm})\end{array}$ & 2.48 & 1.48 & 0.001 \\
\hline $\begin{array}{l}\text { Hormonal status } \\
\text { positive }\end{array}$ & $148(n=200)$ & $264(n=336)$ & 0.225 \\
\hline HER-2 positive & $42(n=180)$ & $59(n=278)$ & 0.503 \\
\hline Tumor grade & & & 0.002 \\
\hline 1 & $22(10.52 \%)$ & $82(23.03 \%)$ & \\
\hline 2 & $85(40.67 \%)$ & 134 (37.64\%) & \\
\hline 3 & $76(36.36 \%)$ & 107 (30.06\%) & \\
\hline Unknown & $26(12.44 \%)$ & $33(9.27 \%)$ & \\
\hline Tumor stage & & & $<0.001$ \\
\hline 0 & 19 (9.09\%) & $36(10.11 \%)$ & \\
\hline 1 & 40 (19.14\%) & 149 (41.85\%) & \\
\hline 2 & 113 (54.07\%) & 148 (41.57\%) & \\
\hline 3 & $36(17.22 \%)$ & 20 (5.62\%) & \\
\hline Unknown & $1(0.48 \%)$ & $5(0.84 \%)$ & \\
\hline $\begin{array}{l}\text { Disease-free } \\
\text { survival (months) }\end{array}$ & 54 & 65 & 0.9950 \\
\hline $\begin{array}{l}\text { Overall survival } \\
\text { (months) }\end{array}$ & 59 & 72 & 0.9988 \\
\hline
\end{tabular}

patients who had undergone BCS was smaller, $1.48 \mathrm{~cm}$, compared to patients who had undergone mastectomy, $2.48 \mathrm{~cm}$, $(P=0.001)$, while there was a higher inclination for BCS over mastectomy in patients with all tumor grades, BCS was more common in earlier stages with increasing preference for mastectomy at higher tumor grades $(P=0.002)$.

Based on matched baseline characteristics, the outcomes of the treatment of mastectomy and BCS for early-stage breast cancer treatment were not significantly different for both months of disease-free survival and overall survival, with statistical $P$ values of 0.9950 and 0.9988 , respectively. The results were also verified using Cox-Breslow regression, which determined that the disease-free survival and overall survival in months between BCS and Mastectomy were not statistically different with $P$ of 0.11 and 0.77 , respectively.

\section{Discussion}

Previous randomized controlled trials conducted to evaluate the outcomes of BCS compared to mastectomy have found no differences in the disease-free survival and overall survival of patients [2-7]. In fact, observational real-world studies using data obtained from national cancer registries have determined that better cancer-specific survival and overall survival outcomes were attained in patients who underwent BCS compared to mastectomy [2, 11-14]. However, in Thailand, while there has been a rise in the incidence of breast cancer, preference for BCS remains very low. As a result, patients are limited to mastectomy for the treatment of early-stage breast cancer culminating with the full loss of breasts and no increased overall treatment outcomes. The disinclination toward BCS may stem from numerous factors including the fear of cancer recurrence with non-full removal of breast tissue as well as the lack of expertise in conducting the surgery $[15,16]$.

In this study, the determinants in the selection of BCS or mastectomy for the treatment of early-stage breast cancer and the outcomes of the treatments were evaluated. In order to evaluate the significance of each particular determinant in the effect on treatment selection without being confounded by other determinants, as well as to compare the outcomes of the treatment without biases from confounding factors, propensity score matching was utilized.

Based on propensity score-matched analysis, the results found no significant differences in patient treatment outcomes comparing BCS and mastectomy, including months of a cancer-free status and overall survival posttreatment of early-stage breast cancer. The results suggest that mastectomy is not superior to BCS and in fact, there is no advantage in BCS compared to mastectomy for the treatment of early-stage breast cancer. Evaluation of the selection of treatment found that the disease characteristics that were statistically significant in affecting the treatment choice were the average clinical tumor size, the tumor grade, and the cancer stage. BCS was more likely to be the treatment choice in patients with smaller tumor size, lower tumor grade, and earlier cancer stages. The significant patient factor that was found to affect the treatment decision was patient age, with younger patients more likely to be treated with BCS, while patients more than 71 were more 
likely to be treated with mastectomy. The results also indicated that physician factors, in particular, the surgeon's treatment preference had a very significant effect on the treatment choice of BCS or mastectomy. All surgeons had a preference for a particular treatment and as a result, would perform the preferred treatment for the majority of their patients.

\section{Conclusion}

This retrospective cohort study reported comparable clinical outcomes between BCS and mastectomy after adjusted for potential confounders, using propensity score matching. The key determinants affecting the selection of treatment were identified to be patient age, characteristics of the tumor, and surgeon's preference.

Author contributions. MW and KP made substantial contributions to the conception and design of the study. MW and $\mathrm{KC}$ substantially contributed to the acquisition of data. MW and KP analyzed and interpreted the data. MW and KP drafted the manuscript and $\mathrm{KC}$ critically revised it. MW, KP, and $\mathrm{KC}$ approved the final version submitted for publication and take responsibility for the statements made in the published article.

Acknowledgments. The authors would like to thank the study nurses for their logistical support, especially Mrs. Bencharat Thumpreechapong for her dedicated data collection effort, as well as Mrs. Aniqa Islam Marshall for the academic support.

Conflict of interest statement. The authors have completed and submitted the International Committee of Medical Journal Editors Uniform Disclosure Form for Potential Conflicts of Interest. None of the authors disclose any conflict of interest.

\section{References}

[1] Ferlay J, Soerjomataram I, Dikshit R, Eser S, Mathers C, Rebelo M, et al. Cancer incidence and mortality worldwide: sources, methods and major patterns in GLOBOCAN 2012: Globocan 2012. Int J Cancer. 2015; 136:E359-86.

[2] Arriagada R, Lê MG, Guinebretière JM, Dunant A, Rochard F, Tursz T. Late local recurrences in a randomised trial comparing conservative treatment with total mastectomy in early breast cancer patients. Ann Oncol. 2003; 14:1617-22.

[3] Blichert-Toft M, Nielsen M, Düring M, Møller S, Rank F, Overgaard $\mathrm{M}$, et al. Long-term results of breast conserving surgery vs mastectomy for early stage invasive breast cancer: 20 -year follow-up of the Danish randomized DBCG-82TM protocol. Acta Oncol. 2008; 47:672-81.
[4] Fisher B, Anderson S, Bryant J, Margolese RG, Deutsch M, Fisher ER, et al. Twenty-year follow-up of a randomized trial comparing total mastectomy, lumpectomy, and lumpectomy plus irradiation for the treatment of invasive breast cancer. N Engl J Med. 2002; 347:1233-41.

[5] Poggi MM, Danforth DN, Sciuto LC, Smith SL, Steinberg SM, Liewehr DJ, et al. Eighteen-year results in the treatment of early breast carcinoma with mastectomy versus breast conservation therapy: the National Cancer Institute Randomized Trial. Cancer. 2003; 98:697-702.

[6] van Dongen JA, Voogd AC, Fentiman IS, Legrand C, Sylvester RJ, Tong D, et al. Long-term results of a randomized trial comparing breast-conserving therapy with mastectomy: European Organization for Research and Treatment of Cancer 10801 trial. J Natl Cancer Inst. 2000; 92:1143-50.

[7] Veronesi U, Cascinelli N, Mariani L, Greco M, Saccozzi R, Luini A, et al. Twenty-year follow-up of a randomized study comparing breast-conserving surgery with radical mastectomy for early breast cancer. N Engl J Med. 2002; 347:1227-32.

[8] Acharya S, Michalski JM, Perkins SM. Distance to nearest radiation facility and treatment choice in early stage breast cancer. J Clin Oncol. 2015; 33:73.

[9] Gu J, Groot G, Boden C, Busch A, Holtslander L, Lim H. Review of factors influencing women's choice of mastectomy versus breast conserving therapy in early stage breast cancer: a systematic review. Clin Breast Cancer. 2018; 18:e539-54.

[10] National Comprehensive Cancer Network. NCCN clinical practice guidelines in oncology: breast cancer [Internet] 2019. [cited 2019 May 1]. Available from: https://www.nccn.org/professionals/ physician_gls/recently_updated.aspx

[11] Chen K, Liu J, Zhu L, Su F, Song E, Jacobs LK. Comparative effectiveness study of breast-conserving surgery and mastectomy in the general population: a NCDB analysis. Oncotarget. 2015; 6:40127-40.

[12] Hartmann-Johnsen OJ, Kåresen R, Schlichting E, Nygård JF. Better survival after breast-conserving therapy compared to mastectomy when axillary node status is positive in early-stage breast cancer: a registry-based follow-up study of 6387 Norwegian women participating in screening, primarily operated between 1998 and 2009. World J Surg Oncol. 2017; 15:118.

[13] Hwang ES, Lichtensztajn DY, Gomez SL, Fowble B, Clarke CA. Survival after lumpectomy and mastectomy for early stage invasive breast cancer: the effect of age and hormone receptor status. Cancer. 2013; 119:1402-11.

[14] van Maaren MC, de Munck L, de Bock GH, Jobsen JJ, van Dalen T, Linn SC, et al. 10 year survival after breast-conserving surgery plus radiotherapy compared with mastectomy in early breast cancer in the Netherlands: a population-based study. Lancet Oncol. 2016; 17:1158-70.

[15] Fisher CS, Martin-Dunlap T, Ruppel MB, Gao F, Atkins J, Margenthaler JA. Fear of recurrence and perceived survival benefit are primary motivators for choosing mastectomy over breastconservation therapy regardless of age. Ann Surg Oncol. 2012; 19:3246-50.

[16] Hershman DL, Buono D, Jacobson JS, McBride RB, Tsai WY, Joseph KA, et al. Surgeon characteristics and use of breast conservation surgery in women with early stage breast cancer. Ann Surg. 2009; 249:828-33. 